\title{
Bundle for pediatric COVID-19 sepsis
}

\author{
Jaime Fernández-Sarmiento ${ }^{*}$ * (D), Werther Brunow de Carvalho²
}

\section{SUMMARY}

COVID-19 infection can progress to severe respiratory infection and have high mortality rates. Several pathophysiological factors are observed in fatal cases, with mortality related to multiple organ failure, in addition to the evolution with high levels of serum ferritin, D-dimer, and C-reactive protein. These severe cases often meet the criteria for macrophage activation syndrome with changes in the host's inflammatory response and an inadequate resolution phase. In the present study, the bundle for COVID-19 sepsis is proposed, including early recognition; protection, handwashing and isolation measures; oxygen therapy; early invasive mechanical ventilation; treatment aimed at modifying the clinical course. This strategy may be useful in the control of children with severe COVID-19 cases, as already demonstrated with the implementation of bundles in sepsis and other etiologies.

KEYWORDS: COVID-19. Sepsis. Infections. Respiratory Distress Syndrome. Respiratory tract infections.

\section{INTRODUCTION}

Several pathophysiological factors have been found in COVID19 fatal cases, which may explain its severe behavior ${ }^{1}$. People with greater comorbidities ${ }^{4,5}$, such as heart disease, diabetes, hypertension, or obesity have been noted to present a greater risk of death. Mortality has been related to multiple organ failure as the common final pathway of pneumonia, sepsis, and acute respiratory distress syndrome (ARDS) (Figure 1).

Patients with this outcome have been found to have higher serum ferritin, D-dimer, and C-reactive protein (CRP) levels than those who survive ${ }^{5}$. These findings have been seen in other severe viral infections, especially in children, due to the activation of some signaling pathways by increased interleukin 6 (IL-6), interleukin 1 (IL-1), and tumor necrosis factor alpha (TNF- $\alpha)^{6}$. These severe cases often meet the criteria for macrophage activation syndrome (MAS), which is characterized by a disorderly inflammatory response in the host with an inadequate resolution phase. One of the defining criteria for MAS has been ferritin $>500 \mathrm{mg} / \mathrm{dL}$, which has also been seen in patients with severe COVID-19 infection ${ }^{5,7,8}$.
This virus, in a septic patient scenario, can release damage-associated molecular patterns (DAMP) and pathogen-associated molecular proteins (PAMPs), which lead to severe inflammation with microcirculation injury, thrombosis, and fibrinogen consumption, coinciding with the increased D-dimer seen in patients who die from COVID-195,9. If this inflammation could be modulated, the microcirculation damage (especially endothelial cell damage) would stabilize, which would stop the apoptotic and pyroptotic cascade, allowing recovery of innate immunity and lung function (the lung being one of the most severely affected organs) ${ }^{10}$.

Accordingly, the implementation of a rapid approach has been a determining factor in many of the outcomes seen in this pandemic ${ }^{1}$, as proposed in the paper by Al-Hajjar and McIntosh $^{5}$, the disease manifestation is less serious than so in the pediatric population than in adults. Thus, a sepsis bundle is proposed for patients with COVID-19 infection, which we believe could be useful in modifying the clinical course of the disease, just as it has proven useful in sepsis from other etiologies $^{11}$ (Figure 2).

\footnotetext{
'Universidad de la Sabana, Fundación Cardioinfantil-Instituto de Cardiologia, Departament of Pediatric Critical Care Medicine - Bogotá, Colombia. ¿Universidade de São Paulo, Instituto da Criança, Pediatrics Department - São Paulo (SP), Brazil.

*Corresponding author: jaimefe@unisabana.edu.co

Conflicts of interest: The authors declare there are no conflicts of interest. Funding: None.

Received on August 20, 2020. Accepted on September 20, 2020.
} 
The bundle components for COVID-19 sepsis should include:

1. Early recognition: The recent sepsis guidelines for both adults and children, as well as the recommendations for management of COVID-19 sepsis, indicate that this measure is key in modifying the clinical course. It should be adhered to fulfill the case definition proposed by WHO, adapted to each center ${ }^{1}$. Keep in mind that fever associated with rapidly developing respiratory

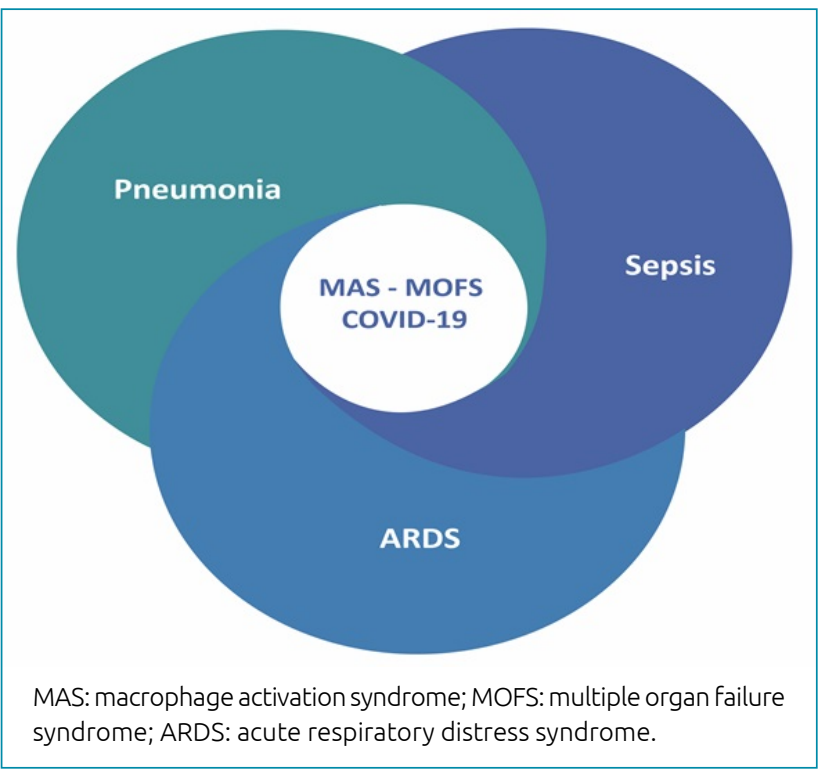

Figure 1. Various pathways leading to MAS and MOFS in COVID-19 infection.

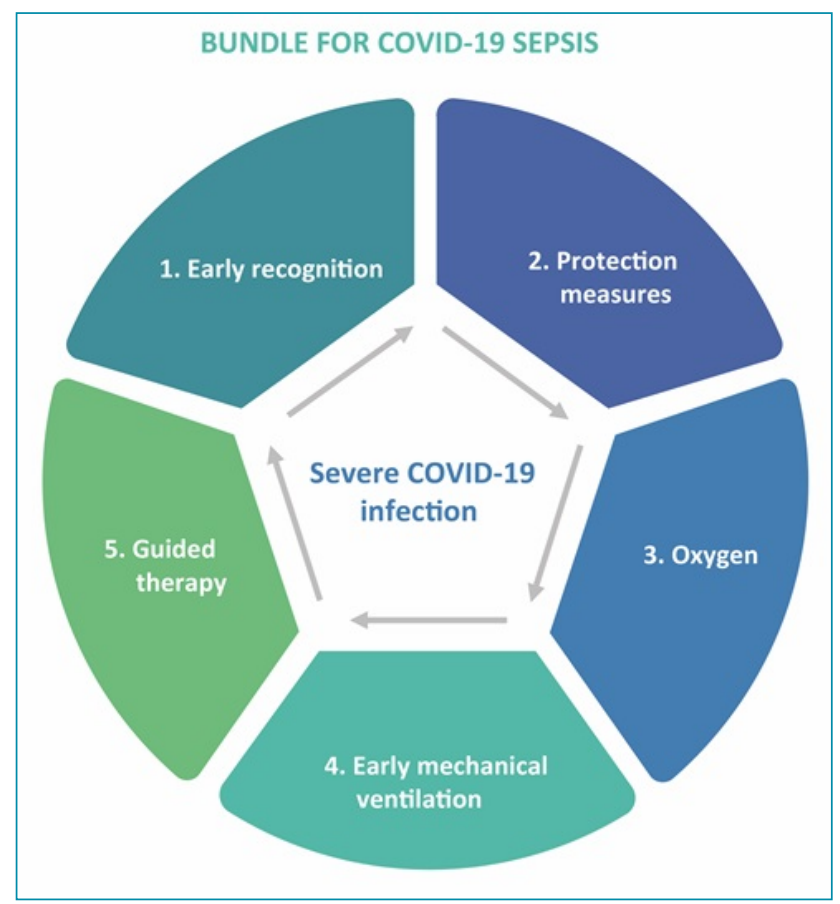

Figure 2. Bundle for COVID-19 sepsis symptoms and deterioration have been the most important characteristic of this pandemic.

2. Protection, handwashing, and isolation measures: These measures are what have helped lower the pandemic's peak. Personal protection for health care personnel who care for these patients is essential. Countries like Spain have described up to $12 \%$ contagion of the health care team ${ }^{1}$. Universal isolation measures will avoid nosocomial contagion and dissemination of the virus (SARS-CoV-2).

3. Oxygen therapy: Given that the lung is the main organ affected, oxygen delivery systems should be offered quickly, according to the patient's needs. Consider using non-rebreather masks with high-efficiency face masks (N95 or FFP) over them ${ }^{1}$. This technique has also been described with the use of high-flow nasal cannulas (HFNCs) to avoid aerosol dissemination ${ }^{2,10}$. The noninvasive ventilation (NIV) should be limited and recommended only if adequate levels of staff protective equipment is available.

4. Early invasive mechanical ventilation: In the experience of the most severely affected countries (China, Italy, and Spain, given their greater number of cases), rapid initiation of mechanical ventilation has been essential for those who have survived ${ }^{1,2}$. Delaying intubation and ventilatory support has been associated with greater mortality ${ }^{5}$. Adequate preparation for intubation, pre-oxygenation without positive pressure ventilation, and rapid sequence of intubation using videolaryngoscope should be considered ${ }^{1,2}$. The presenting pathophysiological pattern will guide the ventilatory support which will cause the least alveolar damage. It may be useful to establish an "optimal" PEEP graphically, numerically or using an esophageal balloon with monitoring with volumetric capnography ${ }^{12}$. The hypoxic pulmonary vasoconstriction phenomenon may be leading to severe pulmonary hypertension, right heart failure, and death. Therefore, reports have indicated that the early use of nitric oxide could be useful. The prone position should be considered when Pa02/Fi02 is $<150^{12}$.

5. Treatment aimed at modifying the clinical course: The studies published to date have not described a specific treatment which could be universally recommended. The use of hydroxychloroquine, azithromycin, and ritonavir, among others, has been reported with inconsistent results ${ }^{13}$. In this respect, the presenting immunophenotype in the disease's severe phase could help guide treatment. If we posit that the scenario meets the MAS criteria with immunoparalysis 
related to MOF (TNF-alpha <200pg / ml), immunoglobulins or colony stimulating factor could be useful ${ }^{8,9}$. Likewise, in patients with TAMOF $(<57 \%$ ADAMTS-13 activity), plasmapheresis, or eculizumab could be considered?

\section{CONCLUSION}

The bundle for COVID-19 sepsis can be a useful strategy for the control of the disease, as it has been demonstrated in sepsis of another etiology. Studies are needed to confirm its utility and its potential to modify the clinical course in this disease.

\section{TO EDITOR}

In December 2019, for the first time in history, a new viral infection was reported, causing severe respiratory infection and extremely high mortality rates ${ }^{1}$. The virus, which based on its genetic sequence belongs to the Betacoronavirus genus, has been found to be highly related to the SARS virus and is known as SARS-CoV-2 (COVID 19) ${ }^{2}$. We have observed its epidemiological behavior with concern, noting that its main characteristic has been its high transmissibility, severity, and lethality, particularly in people over the age of $60^{3,4}$. We have read with great interest the article published in your magazine entitled: "Pediatric COVID-19: An Update on the Expanding Pandemic" by Al-Hajjar and McIntosh'. It is with great interest we see that this pandemic is progressing rapidly, and these papers updates are necessary in pathophysiological, diagnostic, and treatment aspects.

\section{REFERENCES}

1. World Health Organization. Coronavirus disease 2019 (COVID19): situation report. Geneva: World Health Organization; 2020. [cited on August 2, 2020]. Available from: https://www.who.int/ emergencies/diseases/novel-coronavirus-2019/situation-reports

2. Yang $X, Y u$ Y, Xu J, Shu H, Xia J, Liu H, et al. Clinical course and outcomes of critically ill patients with SARS-CoV-2 pneumonia in Wuhan, China: a single-centered, retrospective, observational study. Lancet Respir Med. 2020;8(5):475-81. PMID: 32105632

3. Guan WJ, Ni ZY, Hu Y, Liang WH, Ou CQ, He JX, et al. Clinical characteristics of coronavirus disease 2019 in China. N Engl J Med. 2020;382(18):1708-20. https://doi.org/10.1056/NEJMoa2002032

4. Zhou F, Yu T, Du R, Fan G, Liu Y, Liu Z, et al. Clinical course and risk factors for mortality of adult inpatients with COVID-19 in Wuhan, China: a retrospective cohort study. Lancet. 2020;395(10229):105462. https://doi.org/10.1016/S0140-6736(20)30566-3

5. Al-Hajjar S, Mclntosh K. Pediatric COVID-19: an update on the expanding pandemic. Int J Pediatr Adolesc Med. 2020;7(2):61-3. https://doi.org/10.1016/j.ijpam.2020.05.001

6. Zimmerman O, Rogowski O, Aviram G, Mizrahi M, Zeltser $D$, Justo $D$, et al. C-reactive protein serum levels as an early predictor of outcome in patients with pandemic $\mathrm{H} 1 \mathrm{~N} 1$ influenza A virus infection. BMC Infect Dis. 2010;10(1):288. https://doi. org/10.1186/1471-2334-10-288

7. Shakoory B, Carcillo JA, Chatahm WW, Amdur RL, Zhao $H$, Dinarello CA, et al. Interleukin-1 receptor blockade is associated with reduced mortality in sepsis patients with features of macrophage activation syndrome: reanalysis of a prior phase III trial. Crit Care Med. 2016;44(2):275-81. https:// doi.org/10.1097/CCM.0000000000001402
8. Lachmann G, Knaak C, Vorderwülbecke G, La Rosée P, Balzer F, Schenk T, et al. Hyperferritinemia in critically ill patients. Crit Care Med. 2020;48(4):459-65. https://doi.org/10.1097/ CCM.0000000000004131

9. Carcillo JA, Halstead ES, Hall MW, Nguyen TC, Reeder R, Aneja R, et al. Three hypothetical inflammation pathobiology phenotypes and pediatric sepsis-induced multiple organ failure outcome. Pediatr Crit Care Med. 2017;18(6):513-23. https:// doi.org/10.1097/PCC.0000000000001122

10. Alhazzani W, Møller MH, Arabi YM, Loeb M, Gong MN,

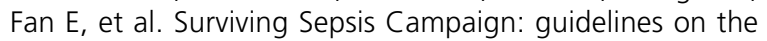
management of critically ill adults with Coronavirus Disease 2019 (COVID-19). Intensive Care Med. 2020;46(5):854-87. https://doi.org/10.1007/s00134-020-06022-5

11. Fernández-Sarmiento J, Carcillo JA, Salinas CM, Galvis EF, López PA, Jagua-Gualdrón A. Effect of a sepsis educational intervention on hospital stay. Pediatr Crit Care Med. 2018;19(6):e321-8. https://doi.org/10.1097/ PCC.0000000000001536

12. Matthay MA, Aldrich JM, Gotts JE. Treatment for severe acute respiratory distress syndrome from COVID-19. Lancet Respir Med. 2020;8(5):433-4. https://doi.org/10.1016/S22132600(20)30127-2

13. Gautret P, Lagier JC, Parola P, Hoang VT, Meddeb L, Mailhe $M$, et al. Hydroxychloroquine and azithromycin as a treatment of COVID-19: results of an openlabel non-randomized clinical trial. Int J Antimicrob Agents. 2020;56(1):105949. https://doi.org/10.1016/j. ijantimicag.2020.105949 